In: Beer, Bettina (Hrsg.): Methoden und Techniken der Feldforschung.

Berlin: Reimer, 2003
Martin Sökefeld

\section{Strukturierte Interviews und Fragebögen}

\author{
Quantitative Methoden in der Ethnologie 95 \\ Zählen: Das Problem der Einheiten 97 \\ Die Standardisierung der Erhebung 99 \\ Die Konstruktion von Fragebögen 100 \\ Sampling 106 \\ Erhebungswege 109 \\ Auswertung 112 \\ Schluss 117 \\ Literatur 117
}

\subsection{Quantitative Methoden in der Ethnologie}

Viele Ethnologen verstehen ihre Wissenschaft als eine Disziplin, die überwiegend mit qualitativen Methoden arbeitet. Teilnehmende Beobachtung und offene Interviews werden als die Standardmethoden der Ethnologie angesehen. Strukturierte Interviews, die mit Fragebögen erhoben und quantitativ ausgewertet werden, gelten dagegen als Methoden der Soziologie und als , unethnologisch“. Der Gegensatz von qualitativen und quantiativen Methoden hat oft eine fast identitätsstiftende Funktion für Ethnologen. Zum Teil werden auch bestimmte theoretische Richtungen der Ethnologie auf den einen oder den anderen Methodenbereich festgelegt. Dann heißt es etwa, analytische Ethnologie arbeite quantitativ, interpretative Ethnologie verwende dagegen qualitative Methoden.

Dieser strikte Gegensatz, bzw. die hinter diesem Schematismus oft verborgene Ablehnung quantitativer Methoden, ist jedoch zu kurz gedacht (Schweizer 1998; Sobo/de Munck 1998). Ethnologen arbeiten sowohl qualitativ als auch quantitativ. Bernard hält fest: "No method of data collection is perfect. Unstructured interviews and questionnaires produce different kinds of data, and it is up to you to decide which method, or combination of methods, is best." (Bernard 1995: 287) 
Tatsächlich kommt keine ethnologische Studie ohne Quantifizierungen aus. Quantitative Methoden haben mit Häufigkeits- und Mengenangaben zu tun. Wenn jede Ethnographie auch quantitative Aspekte hat, bedeutet das natürlich nicht, dass ethnographische Monographien stets ausgefeilte Tabellen und Statistiken enthalten. Sehr oft bleiben Quantifizierungen implizit, vage und damit ungenau. Es heißt dann etwa über die Nuer: "Families often change their place of residence from one part of a village to another (...)" (EvansPritchard 1969: 65). Oder, zu Beginn dieses Beitrags: "Viele Ethnologen..." Wir erfahren nicht genau, wie oft Nuer umziehen, oder für wie viele Ethnologen tatsächlich diese Aussage gilt. Eine genaue Quantifizierung ist vielleicht auch gar nicht nötig - es kommt eben vor allem darauf an, auszudrücken, dass Nuer überhaupt immer wieder umziehen, dass sie nicht dauerhaft am selben Ort wohnen. Aber manchmal wäre eine genauere Zahlenangabe doch sinnvoll. Dies ist vor allem dann der Fall, wenn aus der Zahl der Umzüge weitere Schlüsse gezogen werden sollen, oder wenn Vergleiche angestellt werden zwischen Haushalten, die oft umziehen und solchen, die sesshafter sind.

Quantiative Methoden haben zum Ziel, exakte Mengenangaben zu erreichen. Sie implizieren Zählungen und Messungen. Damit diese Messungen gültig sind, müssen sie mit verlässlichen Messinstrumenten erhoben werden. Standardisierte Erhebungsinstrumente sind erforderlich, die sicherstellen, dass allen Befragten dieselben Fragen gestellt werden, damit die Antworten auch tatsächlich ausgezählt, verglichen, und zu einander in Beziehung gesetzt werden können. Wenn Interviewdaten quantitativ ausgewertet werden sollen, muss also mit strukturierten Interviews gearbeitet werden, die in der Regel mit Hilfe von Fragebögen geführt werden. Bei offenen oder unstrukturierten Interviews gibt der Ethnologe häufig den Gesprächsfaden zu einem gewissen Grad aus der Hand und lässt seine Gesprächspartner (mit-)bestimmen, über welche Themen gesprochen wird. Bei strukturierten Interviews ist dagegen der Ablauf des Gesprächs vorgegeben. Das heißt, sowohl der Wortlaut der Fragen als auch ihre Reihenfolge wird festgelegt und bleibt für alle Gesprächspartner, die bei der Erhebung befragt werden, gleich. Diese Strukturierung und Standardisierung ist erforderlich, um valide Quantifizierung zu ermöglichen.

Die wichtigste Voraussetzung für Zählen und Messen sind definierte Einheiten. Die Definition solcher Einheiten ist in der Ethnologie häufig sehr schwierig. Damit werde ich mich im folgenden Abschnitt auseinandersetzen. Im Weiteren behandelt dieses Kapitel die Standardisierung von Erhebungen, die Konstruktion von Fragebögen, die Auswahl von Informanten (das Sampling) und verschiedene Erhebungswege. Mein Beitrag schließt mit einem kurzen Einblick in die Datenauswertung.

\subsection{Zählen: Das Problem der Einheiten}

Zählen ist einfach, man lernt es spätestens in der Grundschule. Schwierig wird Zählen in der Ethnologie dadurch, dass wir etwas zählen und uns sehr genau darüber klar werden müssen, was wir da zählen. Wir haben es eben nicht - wie in der Mathematik - mit abstrakten Zahlen zu tun, sondern mit konkreten Dingen, Personen oder Ereignissen, deren Anzahl uns interessiert. Wir wollen etwas zählen, und dazu muss zunächst definiert werden, was dieses Etwas ist. Wir stehen vor der Frage der Einheiten des Zählens. Auch das erscheint zunächst vielleicht unproblematisch. Wenn wir wissen wollen, wie viele Menschen in einem Dorf leben, dann zählen wir diese Menschen. Die Einheit ist definiert als das menschliche Individuum. Aber bei genauerem Nachdenken wird klar, dass diese Definition ungenau und keineswegs eindeutig ist. Denn welche Menschen leben in unserem Dorf? Wie lange etwa muss man sich in dem Dorf aufhalten, damit man zur Bevölkerung gezählt wird? Gehört ein Kind dazu, das ein halbes Jahr bei seinen Großeltern im Dorf lebt, ansonsten aber mit seinen Eltern in der Stadt wohnt? Was ist mit Migranten, die sich mal im Dorf aufhalten, mal woanders? Um eine genauere Definition dessen, was gezählt werden soll, kommt man nicht herum. Das Ziel der Definition ist eine klare und eindeutige Abgrenzung dessen, was un ter eine Kategorie gezählt wird, von dem, was nicht dazu gerechnet wird.

Die Definition einer Einheit ist zunächst ein willkürlicher Akt. Ich könnte beispielsweise festlegen: Ein Bewohner des Dorfes ist ein Mensch, der sich mindestens die Hälfte des Jahres in diesem Dorf aufhält. Vielleicht habe ich Aufzeichungen darüber, wer sich wie lange im Dorf aufhält, dann kann ich objektiv sagen, wer unter diese Definition fällt und wer nicht (vermutlich habe ich solche Aufzeichnungen aber nicht, dann ist es schwierig, diese Definition anzuwenden).

Trotzdem kann es sein, dass diese Definition nicht besonders sinnvoll ist. Vielleicht fallen Individuen heraus, die nicht die Hälfte des Jahres im Dorf leben, die aber im dörflichen sozialen Gefüge eine wichtige Rolle spielen und von den anderen Dorfbewohnern als Einwohner betrachtet werden. Was ist dann wichtiger, unsere , objektive“ Definition, oder die Ansicht der Dorfbewohner? Wir müssen uns zwischen einer emischen und einer etischen Definition entscheiden. Über diese beiden aus der Linguistik übernommenen Konzepte hat es in der Ethnologie eine langandauernde Debatte gegeben (Headland et al. 1990). Grob gesagt bezieht sich emisch auf das Bedeutungsuniversum der jeweils Untersuchten, während etisch Kategorien und Definitionen meint, die vom Forscher von außen an den Untersuchungsgegenstand herangetragen werden. Meine selbst festgelegte Definition wäre also etisch, die Defini- 
tion von ,Bewohner' durch die Einwohner des Dorfes selbst wäre dagegen eine emische Definition.

Die Unterscheidung von emischer und etischer Perspektive erleichtert es, die Problematik der Definition von Einheiten der Zählung zu veranschaulichen, aber sie löst diese Schwierigkeit nicht. Häufig gibt es nämlich nicht, die‘ emische Perspektive in einer untersuchten Gruppe, sondern verschiedene, konfligierende Sichtweisen. Neben unterschiedlichen lokalen Ansichten mag es auch noch eine offizielle Definition des ,Einwohners ‘ durch die staatliche Verwaltung geben, die sich von den lokalen Definitionen unterscheiden kann. Bei meiner Feldforschung in Gilgit/Nordpakistan, stellte sich beispielsweise heraus, dass es höchst umstritten war, wer im Ort als Einwohner galt und wer nicht. Das Konzept des ,Einwohners ' umfasste nämlich eine Reihe von Rechten auf Nutzung knapper Ressourcen (vor allem Land und Wasser), die keineswegs allen Bewohnern zugestanden wurden. Aber es gab keine Einigkeit darüber, wer nun Einwohner war und wer nicht. Manche Familien, die von einigen als Einwohner anerkannt wurden, wurden von anderen als, Leute von außen' kategorisiert, die keine Einwohner sind und keine Rechte haben. Der Rückgriff auf die offizielle Definition der Verwaltung brachte hier keine Lösung, denn manche Einwohner warfen der Verwaltung Korruption vor: Sie habe den Status des Einwohners auch ,Leuten von außen" eingeräumt, die kein Recht darauf haben. Man muss sich darüber im Klaren sein, dass in einer solchen Situation der einfache Forschungsakt des Zählens ein politischer Akt sein kann, der die Version einer bestimmten Fraktion in der untersuchten Gruppe stützt und selbst zum Teil des Konfliktes werden kann.

Ein zweites Beispiel für die Problematik der Definition von Einheiten der Befragung: Bei ihrer Untersuchung in Dörfern im nordpakistanischen Bagrot wollte Monika Schneid (persönl. Mitteilung) auch Daten über den Viehbesitz der Haushalte sammeln. Sie fragte jeweils, wie viele Kühe und Ziegen ein Haushalt besaß. Erst im Nachhinein stellte sie fest, dass sie mit dieser Frage nicht einfach den Viehbestand der Dörfer erhoben hatte. Vor allem Kühe hatte sie mehr gezählt, als tatsächlich vorhanden waren. Denn die Dorfbewohner praktizierten bei Kühen, die eine teure Investition darstellten, kollektiven Viehbesitz. Dieses, Cowsharing ' bedeutete, dass eine Kuh mehrere Besitzer haben konnte. Bei der Befragung hatte aber jeder der (Teil-)Besitzer die Kuh als seinen Besitz genannt, so dass Tiere zum Teil mehrfach gezählt worden waren.

Wie viele Einwohner ein Dorf hat oder wie viele Kühe jemand besitzt sind also nicht unbedingt Fragen, die sich, objektiv" beantworten lassen. Die Beantwortung dieser Fragen ergibt sich nicht einfach, aus der Empirie‘. Was die jeweilige Empirie ist, hängt davon ab, wie wir die Fragen formulieren und wie sie von unseren Gesprächspartnern verstanden werden. Wenn man in die Tiefe geht, wird man für sehr viele auf den ersten Blick einfache Fragen feststellen, dass sie nicht so einfach zu beantworten sind, wie sie ursprünglich zu sein scheinen, bzw., dass in ihre Beantwortung zahlreiche Vorannahmen flieBen, die nicht unmittelbar offensichtlich sind. Wenn man die Befragung aufgrund dieser Schwierigkeiten nicht gleich wieder aufgeben will, dann muss man sich pragmatisch für eine Definition der jeweils in Frage stehenden Kategorie entscheiden. Welche Definition sinnvoll ist, muss aus dem Forschungskontext heraus entschieden werden, dafür kann es keine allgemeine Lösung geben.

\subsection{Die Standardisierung der Erhebung}

Aus der Diskussion um die Definition der Einheiten ergibt sich die entscheidende Forderung: Wie auch immer die jeweilige Einheit definiert wird, diese Definition muss für die ganze Erhebung durchgehalten werden. Die Verwendung verschiedener Definitionen in ein und derselben Untersuchung führt $\mathrm{zu}$ inkonsistenten Daten, die nicht untereinander vergleichbar sind und aus denen daher keine gültigen Schlüsse gezogen werden können.

Die Standardisierung dient dazu, die Vergleichbarkeit der erhobenen Daten herzustellen. So soll ein Fragebogen sicherstellen, dass allen Informanten dieselben Fragen gestellt werden und dass sie ihnen auf dieselbe Art und Weise gestellt werden. Der zweite Aspekt ist natürlich nicht vollständig zu erfüllen, denn die Art und Weise des Fragens kann auch von so unkontrollierbaren Bedingungen wie dem Wetter oder der momentanen psychischen Verfassung des Interviewers beeinflusst werden. Die Bedeutung einer Frage ergibt sich schließlich nicht nur aus ihrem Wortlaut sondern etwa auch aus ihrer Betonung. Tatsächlich kann ein Fragebogen also nur gewährleisten, dass die Unterschiede der Art und Weise, wie dieselben Fragen verschiedenen Gesprächspartnern gestellt werden, minimiert werden.

Das strukturierte Interview ist eindeutig vom Forscher dominiert. Der jeweilige Gesprächspartner hat auf die Themen, die besprochen werden, keinen Einfluss. Er kann lediglich die Beantwortung von Fragen verweigern. Dies ist ein wichtiger Punkt, denn er besagt, dass durch strukturierte Interviews selbst keine neue Fragestellung für die Untersuchung entwickelt und nichts über die Bedeutung der gestellten Fragen für die Forschung erfahren werden kann. Was als wichtig angesehen wird und was nicht, ist durch die Konstruktion des Fragebogens bereits festgelegt. 
Daraus folgt eine wichtige Konsequenz für den Einsatz strukturierter Interviews und Fragebögen im Rahmen einer Feldforschung: Die Konstruktion eines sinnvollen Fragebogens erfordert bereits erhebliches Wissen über die untersuchte Gruppe und das Thema, das erforscht werden soll. Eine standardisierte Befragung ist also keine Methode, mit der man sich einem neuen, weitgehend unbekannten Untersuchungsfeld nähern sollte. Dies gilt auch aus Gründen der Forschungspragmatik: Es ist so aufwändig, einen Fragebogen $\mathrm{zu}$ erstellen und eine standardisierte Befragung durchzuführen, dass dafür vorhandene Ressourcen sehr überlegt und zielgenau eingesetzt werden sollten. Vorwissen ist dabei in mindestens zweierlei Hinsicht erforderlich: Einerseits muss schon bekannt sein, welche Fragen im Kontext der Forschung tatsächlich relevant sind, andererseits muss man wissen, wie man die gewünschten Informationen erreichen kann, d. h. wie die beabsichtigten Fragen gestellt werden müssen.

\subsection{Die Konstruktion von Fragebögen}

Die Konstruktion eines Fragebogens sowie die Art des notwendigen Vorwissens hängt davon ab, welche Art von Informationen mit ihm erhoben werden sollen. Geht es etwa um allgemeine Daten, die eine Bevölkerung beschreiben, wie Alter, Geschlecht, Wohnort, Familienstand, Zahl der Kinder usw.? Viele solcher Fragen benötigen eher geringes Vorwissen, da die Dimensionen einer allgemeinen Beschreibung der untersuchten Gruppe weitgehend vom Untersucher und seinem Interesse festgelegt werden und kaum kulturelle Kategorien in sie eingehen. Aber auch hier benötigt man einen gewissen Forschungsstand, um zu entscheiden, welche dieser Informationen zur Beantwortung übergeordneter Fragen erforderlich sind. Anders ist es bei Befragungen, die auf Wissen über Einstellungen, auf Werte oder kulturelles Wissen, also auf Kognitionen zielen, sowie bei solchen, die nach bestimmten Handlungen fragen. Hier kann das erforderliche Vorwissen sehr umfangreich sein.

\subsubsection{Der Fragebogen als Produkt vorhergehender Forschung}

Wie erfährt man das Wissen, das man benötigt, um einen sinnvollen Fragebogen zu erstellen? In der Regel geht der Konstruktion eines Fragebogens eine längere Phase der Feldforschung voraus, in der man erfährt, welche Aspekte eines Themas wichtig sind, welchen Aspekten von den Angehörigen der untersuchten Gruppe Bedeutung beigemessen wird, wie die Untersuchten über diese Bereiche sprechen (d. h. welche Terminologie und Kategorien sie ver- wenden) und über welche Aspekte es sinnvoll wäre, quantitative Daten zu erheben.

Es kommt also zunächst darauf an, Fragen zu entwickeln, die in den Fragebogen aufgenommen werden sollen. Diese Fragen werden auch Items genannt. Da man auf jede Frage verschiedene Antworten geben können muss (sonst wäre die Frage überflüssig), nennt man die erfragte Information Variable. Das Item, Was ist Ihr Geschlecht?" fragt nach der Variable, Geschlecht ${ }^{*}$ und kann beispielsweise die Ausprägung , weiblich 'oder , männlich“ annehmen. Die meisten Items sind jedoch komplexer und können mehr als nur zwei Merkmalsausprägungen haben.

\subsubsection{Wie viele Fragen? Pragmatische Aspekte}

Zunächst erstellt man also eine Liste der Items, die in einen Fragebogen aufgenommen werden sollen. Dabei ist zu beachten, dass die Beantwortung von Fragebögen Zeit kostet. Ausführliche Fragebögen mit vielen Items kosten viel Zeit, und man kann davon ausgehen, dass das Interesse und die Bereitschaft der meisten Informanten, an einer Befragung teilzunehmen, mit dem erforderlichen Zeitaufwand abnimmt. Susan Weller (1998: 376) rät, lieber zu viele als zu wenige Fragen zu stellen, da es in der Regel unmöglich ist, später noch einmal zu allen Informanten zu gehen und die Fragen zu stellen, die zwar nicht im Fragebogen enthalten waren, die sich aber im Nachhinein als auch noch wichtig entpuppten. Ich halte das nur dann für einen sinnvollen Ratschlag, wenn , zu viele Fragen' nicht einen zu langen Fragebogen ergeben, den niemand mehr beantworten will. Dabei geht es natürlich nicht nur um den Zeitaufwand der Informanten sondern auch um den der Interviewer. Wenn Ressourcen für mehrere Interviewer zur Verfügung stehen, kann in das einzelne Interview mehr Zeit investiert werden, als wenn eine Person sämtliche Interviews führen muss - und daneben auch noch andere Methoden anwenden möchte. Die Zeit, die ein Interview tatsächlich erfordert, ist nicht unbedingt im Voraus absehbar. Sie hängt nicht nur von der Länge des Fragebogens ab, sondern auch davon, ob tatsächlich nur die einzelnen Items durchgegangen werden sollen, oder ob auch Nebenbemerkungen der Informanten aufgezeichnet werden sollen. Manche Informanten antworten knapp und präzise, andere erzählen zu jeder Frage kürzere oder längere Geschichten, die es durchaus auch wert sein können, als Zusatzinformationen festgehalten zu werden.

Aber natürlich hat Weller Recht, dass es ein schwerer Rückschlag für eine Erhebung ist, wenn man nach ihrer Durchführung feststellt, dass eine wichtige Frage nicht gestellt worden ist. Daraus folgt für mich aber weniger die Forderung, gleich so viele Fragen wie irgendmöglich zu stellen, als die, sich 
über Sinn und Zweck der Erhebung im Rahmen der Gesamtforschung sowie über die Gestaltung eines Fragebogen sehr genau Gedanken zu machen und den Fragebogen gut zu testen. Ich denke aber auch, dass es sich zu einem gewissen Grad nicht vermeiden lässt, dass später noch neue Fragen auftauchen. Da Forschung ein offener Prozess ist, der zu neuen Erkenntnissen führen soll, werden ständig neue Fragen enstehen, die dem Forscher zu Beginn nicht in den Sinn gekommen sind. Das gilt besonders für ethnologische Feldforschung, die in der Regel immer auch einen explorativen Charakter hat und somit Aspekte umfasst, die nicht genau im Voraus gesehen werden können. Auch das ist ein Argument dafür, eine standardisierte Befragung erst dann durchzuführen, wenn der Stand der Forschung schon fortgeschritten ist.

\subsubsection{Die Formulierung von Fragen}

Die Frage nach dem Geschlecht bereitet in der Formulierung normalerweise keine Schwierigkeiten. Das trifft leider nicht auf alle Fragen zu, auch wenn sie zunächst ebenso einfach erscheinen. Auch die Frage nach dem Geburtsort bei Migranten scheint zunächst unproblematisch. Wenn man aber beispielsweise türkische Migranten nach ihrem Geburtsort fragt, bekommt man in der Regel nicht wirklich den Ort der Geburt genannt (d. h., ein Dorf oder eine Stadt), sondern die Provinz, in der dieser Ort liegt. Wenn man genauere örtliche Angaben haben will als die Provinz der Geburt, dann muss man die Frage spezifizieren oder erklären, etwa durch den Zusatz, dass nicht (nur) die Provinz gemeint ist, sondern der tatsächliche Ort der Geburt. Welche Fragen einfach sind und welche nicht, bzw. wie eine Frage formuliert werden muss, damit man tatsächlich das erfährt, was man wissen will, das lernt man erst, wenn man die Fragen tatsächlich stellt und ausprobiert.

\subsubsection{Geschlossene Fragen und offene Fragen}

Grundsätzlich können Fragen in einem Fragebogen auf zwei Arten gestellt werden: mit vorgegebenen Antwortmöglichkeiten (geschlossene Fragen) oder ohne vorgegebene Antworten (offene Fragen). Die Frage nach dem Geschlecht, bei der man je ein Kästchen zum Ankreuzen für ,weiblich“ oder ,männlich vorgibt, ist eine geschlossene Frage. Die Frage nach dem Geburtsort, bei der keine Liste möglicher Orte genannt wird, sondern der Befragte selbst den Ort nennen soll, ist eine offene Frage. Man kann jede Frage als offene oder als geschlossene Frage formulieren, aber die Pragmatik wird geschlossene Fragen für manche Bereiche ausschließen. So wäre es wenig sinnvoll, eine Liste möglicher Geburtsorte in der Türkei in den Fragebogen einzufügen, da er dann den Umfang eines mittleren Telefonbuchs hätte. Offene Fragen sind auch dann ratsam, wenn die Antwortmöglichkeiten auf eine Frage nicht im Voraus bekannt sind. Wenn ich also davon ausgehe, dass es in einer Gesellschaft nur zwei Geschlechter gibt, dann kann ich problemlos die Frage nach dem Geschlecht geschlossen formulieren und die beiden Antwortmöglichkeiten vorgeben. Wenn ich aber nicht genau weiß, ob es nicht vielleicht weitere $\mathrm{Ge}$ schlechter gibt, dann muss die Frage offen formuliert werden, da die Geschlossenheit weitere Antworten verhindern würde.

Geschlossene Fragen setzen also voraus, dass alle möglichen Antworten auf die Frage bekannt sind. Die vorgegebenen Antwortmöglichkeiten müssen erschöpfend sein, es darf keine Antwortmöglichkeit geben, die nicht erfasst wird. Und sie müssen wechselseitig ausschließend sein, es muss also eindeutig klar sein, welche der angebotenen Antworten zutrifft. Diese zweite Bedingung bedeutet nicht, dass nur jeweils eine Antwort zutreffend sein darf, da je nach Frage auch Mehrfachantworten möglich sein können. Wenn ich Migranten aus der Türkei nach ihrer Staatsangehörigkeit frage und ,deutsch * und ,türkisch ' als Antwortmöglichkeiten vorgebe, dann kann es sein, dass auf einige Informanten beide Antworten zutreffen, da sie zwei Staatsangehörigkeiten besitzen. Wichtig ist aber, dass die beiden Antwortmöglichkeiten klar voneinander abgegrenzt sein müssen. Wenn etwa in der Türkei nach ethnischer $\mathrm{Zu}$ gehörigkeit gefragt wird und (unter anderem) die beiden Antwortkategorien ,Kurt (Kurde) und ,Zaza ' gegeben werden, so sind diese beiden Antworten nicht klar voneinander abgegrenzt, da oft - aber nicht immer - Zaza als eine Unterkategorie von Kurden verstanden wird. Für einen Zaza-Informanten wäre also nicht klar, ob er beide Antwortmöglichkeiten als auf sich zutreffend betrachten sollte oder nicht, und für den Interviewer ist nicht klar, ob der Informant ,Zaza ' und ,Kurt ‘ als exklusive Kategorien verstanden hat oder nicht.

\subsubsection{Problematische Fragen}

Manche Fragen werden schnell und ohne Vorbehalte beantwortet, anderen weicht man lieber aus. Leider sind die schwierigen Fragen oft gerade die, die uns besonders interessieren. Fragen nach Einkommen oder Besitz etwa, die der Feststellung von Schichtzugehörigkeit und ökonomischem Status dienen, wecken häufig Befürchtungen, die Steuerverwaltung könnte hinter der Befragung stehen oder sich zumindest die Ergebnisse nutzbar machen. Solche Befürchtungen sind nicht immer leicht zu zerstreuen, da man Fragebogenerhebungen ja bei vielen Informanten durchführt und gerade auch bei solchen, denen man nicht aus offenen Interviews oder teilnehmender Beobachtung gut bekannt und vertraut ist. Das Hantieren mit Fragebögen hat immer einen ge- 
wissen ,offiziellen ' Charakter. Viele Informanten kennen Fragebögen vielleicht nur aus staatlichen Zensuserhebungen, bei denen der Zusammenhang zwischen Wissen und Macht bzw. staatlicher Kontrolle unmittelbar auf der Hand liegt. Man denke nur an die Erregung, die die Volkszählungspläne 1987 in Deutschland hervorriefen. Gerade in Ländern mit weniger entwickeltem Datenschutz kann das Misstrauen groß und berechtigt sein. Für die Ergebnisse der Erhebung kann sich Misstrauen verheerend auswirken, weil es in der Regel zu kreativen Verweigerungsstrategien führt, nämlich zu falschen oder irreführenden Angaben. Dies ist für das Ergebnis schlimmer als die direkte Weigerung zu antworten. Nicht gegebene Antworten kann man bei der Auswertung als solche behandeln. Falsche Angaben sind dagegen häufig nicht zu identifizieren. Man kann versuchen, ein gewisses Vertrauensverhältnis zu unbekannten Informanten aufzubauen, indem man sich über geeignete Mittelspersonen einführen lässt. Das wird aber bei einer Zufallsstichprobe in den meisten Fällen unmöglich sein.

Andere Fragen werden vielleicht gerade dann nicht gerne beantwortet, wenn der Interviewer gut bekannt ist. Diese betreffen vor allem intime Lebensbereiche wie Sexualität. Fragen dazu werden manchmal als Bedrohung empfunden. Hierbei ist zu beachten, dass es natürlich kulturell unterschiedlich sein kann, welche Fragen Intimes berühren und damit problematisch sind. Man kann solche Fragen mit einem Satz einleiten, um ihnen etwas von ihrem ,Bedrohungscharakter ${ }^{*}$ zu nehmen. Ich halte jedoch nichts davon, derartige Fragen psychologisch besonders geschickt zu formulieren, um den Informanten eine Antwort zu entlokken, die sie eigentlich nicht geben wollen. Aus Gründen der Forschungsethik finde ich es besser, sich im Zweifelsfall mit ,keine Antwort' zufrieden zu geben.

In jedem Fall setzen beide Kategorien problematischer Fragen voraus, dass den Informanten verlässlich und nachvollziehbar Anonymität und Datenschutz zugesichert wird. Häufig ist es sinnvoll, dies in einer schriftlichen Erklärung zu tun - bei verschickten Fragebögen etwa in einem Begleitschreiben zum Fragebogen. Bei selbst ausgefüllten Fragebögen erfordert Anonymität, dass alle Angaben, die den Informanten identifizierbar machen, vom Hauptteil der Fragen klar getrennt werden.

Schließlich gibt es andere Fragen, die problematisch sind, obwohl sie weder Intimes noch steuerlich Relevantes berühren. Sie betreffen Dinge, deren Quantität nicht genau bekannt ist. So kann in vielen Gesellschaften die Frage nach dem Alter schwierig sein, da die meisten Menschen nicht genau wissen, wann sie geboren wurden. Auch ein Blick in den Ausweis, falls vorhanden, hilft hier nicht unbedingt weiter, da auch er oftmals nur ein geschätztes Geburtsdatum enthält. Früher war es beispielsweise in der Türkei üblich, neugeborene Kinder nur alle paar Jahre (und oft eben längere Zeit nach der Geburt) bei den Behörden anzumelden, mit der Konsequenz, dass die offizellen Daten nicht verlässlich sind. Ähnliche Probleme können sich beim Landbesitz ergeben. Auch hier sind häufig keine genauen Flächenmaße bekannt. Man kann dann versuchen, sich mit Ersatzmaßen zu behelfen, etwa mit der Arbeitszeit, die benötigt wird, um die Felder zu pflügen, oder mit der Menge des Saatgutes, das für die Aussaat erforderlich ist.

\subsection{6 Übersetzungen und Pretests}

In der Regel führen Ethnologen ihre Befragungen nicht in ihrer eigenen Muttersprache durch. Fragebögen und andere Erhebungsinstrumente werden meistens in der Sprache des Ethnologen erstellt und dann in die Sprache der Informanten übersetzt. Aber oft lassen sich Konzepte nicht direkt aus einer Sprache in eine andere übersetzen. Manchmal gibt es in der anderen Sprache kein Wort, dass einen bestimmten Begriff aus der Sprache des Forschers direkt wieder gibt. Homonyme oder Synonyme sind in den beiden Sprachen unterschiedlich, so dass auch dort, wo es direkte Entsprechungen gibt, das jeweilige semantische Feld differieren kann. Oft gibt es aber auch die zentralen Kategorien der Fragen nicht in der Sprache der Ethnologen, wenn es etwa um lokale, emische Konzepte geht. All das erfordert große Sorgfalt bei der Übersetzung von Fragebögen. Ethnologen sollten sich daher nicht auf die eigenen Kenntnisse der Sprache der Informanten verlassen, sondern die Übersetzung von Muttersprachlern oder sogar von ausgebildeten Übersetzern machen lassen. Manchmal ist es sinnvoll, mehrere Übersetzungen in Auftrag zu geben und miteinander abzugleichen. Viele Autoren schlagen vor, dass die Übersetzung wieder in die Sprache des Ethnologen zurück übersetzt wird, um eventuelle Bedeutungsverschiebungen erkennen zu können. Brislin (1986) fordert sogar einen doppelten Durchgang durch diesen Übersetzungskreislauf, um Probleme ausschließen zu können.

Die Schwierigkeit der Auswahl und Formulierung von Fragen sowie der Übersetzung macht es erforderlich, dass der Fragebogen an mehreren Informanten getestet wird, bevor man mit der eigentlichen Untersuchung beginnt. Erst wenn die Fragen tatsächlich Informanten gestellt werden, wird deutlich, wo Bedeutungen unklar sind, wo Fragen missverstanden werden können, wo Einheiten problematisch sind oder sonstige Schwierigkeiten auftauchen. Erst bei einem Pretest wird etwa offensichtlich, welche impliziten Vorannahmen in den Fragen stecken, die von den Informanten nicht geteilt werden. Nach dem Pretest muss der Fragebogen auf der Basis der gewonnenen Erfahrungen gründlich überarbeitet werden. Wichtig ist, dass die Testpersonen nicht aus dem Sample stammen dürfen, das letztendlich befragt werden soll. 


\subsection{Sampling}

\subsubsection{Wer soll befragt werden?}

Will man eine Erhebung in einer kleinen Siedlung oder in einer Nomadengruppe durchführen, die nur wenige Haushalte umfasst, dann kann man tatsächlich alle Mitglieder der Gruppe befragen. Sehr oft, bei fast allen Forschungen, die in Städten durchgeführt werden, ist das jedoch nicht der Fall. Man kann nur einen Teil der Gruppenmitglieder interviewen. In der Regel möchte man mit der Befragung aber zu Daten kommen, die nicht nur etwas über die tatsächlich Befragten aussagen, sondern über die gesamte Gruppe. Dies ist problematisch, weil man davon ausgehen kann, dass die Gruppe nicht homogen ist. Wenn man Gruppenmitglieder A und B befragt hat, kann man nicht schließen, dass $C$ und D dieselben Antworten geben würden. Und wahrscheinlich haben schon A und B unterschiedliche Antworten gegeben. Das Zauberwort heißt hier Repräsentativität: Die Stichprobe von Gruppenmitgliedern (das Sample), die befragt werden, soll repräsentativ für die gesamte Gruppe sein, so dass Aussagen, die über das jeweilige Sample gemacht werden, mit großer Wahrscheinlichkeit auf die gesamte Gruppe zutreffen.

Wie wählt man ein repräsentatives Sample aus? Sampling ist in der Regel eine schwierige Forschungsoperation. Eigentlich müsste man bereits die Gesamtgruppe kennen, um eine Stichprobe mit beispielsweise der gleichen Alters-, Geschlechts-, Einkommensstruktur ziehen zu können, wie sie in der Gesamtgruppe vorherrscht. Da das nicht der Fall ist (denn dann wäre die Erhebung ja überflüssig), behilft man sich mit einem Zufallssample. ,Zufall ' heißt hier nicht, dass die Informanten einfach zufällig ausgewählt werden, denn, zufällig ' in diesem Sinne wäre gleichbedeutend mit, willkürlich“ oder, beliebig'. Zufall bedeutet, dass jedes Mitglied der Gesamtgruppe die selbe Chance haben muss, ausgewählt werden zu können. Es darf also keine Faktoren geben, die bewirken, dass bei einigen Gruppenmitgliedern eine größere Wahrscheinlichkeit besteht, in das Sample aufgenommen zu werden, als bei anderen.

Um ein Zufallssample herstellen zu können, muss man durchaus etwas über die Gesamtgruppe wissen, nämlich wer alles zur Gruppe gehört und in das Sample fallen kann. Man braucht einen Rahmen, ein Sampling frame, aus dem gewählt wird. Ein Sampling frame ist eine Liste, in der alle Mitglieder einer Gruppe verzeichnet sind. Das kann ein Einwohnerregister oder ein Telefonbuch (Bernard 1995: 84) sein, wenn es um eine Studlie in einer Stadt oder einem Stadtteil geht oder etwa die Liste der Schüler einer Schule, wenn diese Schülerschaft untersucht werden soll. Falls kein Sampling frame zu- gänglich ist, muss man selbst einen Rahmen herstellen, etwa, in dem man einen Zensus aufnimmt. Da dies bei einer größeren Gruppe nahezu unmöglich ist, wird man in diesem Fall alle Kreativität daran setzen, doch ein bereits bestehendes Sampling frame zu verwenden.

Nur wenn man einen Rahmen für das Sampling hat, kann man überlegen, wie man vorgehen muss, um daraus eine Zufallsstichprobe auszuwählen. Da jedes Individuum des Sampling frames dieselbe Chance haben muss, ausgewählt werden zu können, hat es sich eingebürgert, mit Tabellen von Zufallszahlen vorzugehen. Eine solche Tabelle findet sich in Bernard (1995: 514ff.). Man nummeriert alle Individuen des Rahmens durch und muss festlegen, wie viele Individuen im Sample enthalten sein müssen (s. u.). Dann geht man die Zufallszahlen von einem beliebigen Einstieg aus der Reihe nach durch und wählt immer ein Individuum aus, wenn dessen Nummer in der Folge der Zufallszahlen vorkommt. Das macht man so lange, bis man so viele Individuen ausgewählt hat, wie das Sample enthalten soll.

Diese Methode ist bei sehr großen Sampling frames impraktikabel, da man nicht alle Mitglieder einer großen Gruppe durchnummerieren kann. Man kann sich dann mit einem Sampling Intervall behelfen. D. h. man beginnt mit einem zufällig ausgewählten Individuum in der Liste des Sampling frame und wählt jedes $n$-te Individuum in der Folge aus, wobei $n$ für das Intervall, den ,Abstand ' zwischen den jeweils ausgewählten Individuen steht. Wenn man bei einer Gruppe von 10000 Individuen jedes 25 . Individuum nimmt, bekommt man ein Sample von 400. Das Intervall ist demnach abhängig von der Größe des Rahmens und des gewünschten Samples. Beim Intervall Sampling muss man darauf achten, dass das Sample nicht durch eine mögliche Regelmäßigkeit der Gesamtgruppe beeinflusst wird. Angenommen, man will in einer großen Reihenhaussiedlung jeden zehnten Haushalt befragen, und die Siedlung ist so aufgebaut, dass jedes zehnte Haus ein Eckhaus mit großem Garten ist. Dann kann es passieren, dass das Sample entweder nur oder gar keine Eckhäuser enthält. Das Sample wäre also nicht repräsentativ. Wenn eine solche Periodizität erkennbar ist, muss man ein Sample-Intervall wählen, das nicht parallel zu dieser Regelmäßigkeit verläuft.

Falls man ein Sample aus einer Gruppe ziehen will, die nach einem bestimmten Kriterium (z. B. Migrationserfahrung) eine zahlenmäßig nicht sehr große, für Vergleichszwecke der Untersuchung aber wichtige Subpopulation enthält, dann kann es sinnvoll sein, für jede der Gruppe ein eigenes Zufallssample zu bilden. Angenommen, zehn Prozent einer Gesamtgruppe von 10000 sind Migranten und man möchte ein Sample von 400 bilden, dann kann man bei der Zufallsauswahl eines Gesamtsamples nicht unbedingt davon ausgehen, 
dass man darunter gerade 40 Migranten (= zehn Prozent) auswählt. Vielleicht enthält das Zufallssample nur 30 Migranten. In diesem Fall ist es besser, gleich zwei Samples zu bilden: eins mit 360 Nicht-Migranten und eins mit 40 Migranten. In diesem Fall hat man zwei proportionale Samples gebildet. Wenn eine Untergruppe, die man erfassen möchte, relativ zur Gesamtbevölkerung sehr klein ist, kann es auch sinnvoll sein, nicht-proportionale Samples zu bilden. Man wählt dann im Vergleich zur Hauptgruppe überproportional viele Angehörige der Untergruppe aus, um zu gewährleisten, dass die Untergruppe im Sample ausreichend vertreten ist.

Falls kein praktikabler Sampling frame zur Verfügung steht, da die zu untersuchende Gruppe zu groß und zu unübersichtlich ist, kann man sich mit Cluster Samples behelfen. Bei einer Stadtforschung unterteilt man etwa die Stadt in Stadtteile, Nachbarschaften oder Häuserblocks (die Cluster), unter denen man ein Zufallssample auswählt. In diesen zufällig ausgewählten Clustern kann dann jeweils ein repräsentatives Sample erstellt werden.

\subsubsection{Wie groß soll ein Sample sein?}

Wir wissen nun, wie man Samples auswählen kann, aber das beantwortet noch nicht die Frage danach, wie groß ein Sample zum Zweck der Untersuchung sein soll. Die notwendige Größe des Samples ist eine Funktion seiner Heterogenität. Wenn alle Individuen in einer Stadt mit einer Million Einwohner völlig identisch wären, dann bräuchte man nur eine Person zu befragen, um Informationen über alle Bewohner zu haben. Derartige extreme Homogenität ist aber bei unseren Untersuchungsgegenständen nicht vorhanden. Da im Voraus nicht bekannt ist, wie homogen oder heterogen eine Gruppe ist, geht man auf Nummer sicher, indem man ihre größtmögliche Heterogenität annimmt. Die Größe des erforderlichen Samples wächst mit der Größe der Gesamtgruppe, über die etwas ausgesagt werden soll. Allerdings wächst die Größe des Samples nicht proportional zur Gesamtgruppe. Im Gegenteil: Für kleine Gesamtgruppen ist ein relativ großes Sample erforderlich, für große Gesamtgruppen aber nur ein relativ kleines Sample. Die Größe des erforderlichen Samples hängt dabei auch von der gewünschten Wahrscheinlichkeit ab, dass das Sample tatsächlich die Gesamtgruppe repräsentiert, d.h. dass die Verteilung eines bestimmten Merkmals im Sample tatsächlich der Verteilung desselben Merkmals in der Gesamtgruppe entspricht. Bernard gibt an, dass bei 95\% Wahrscheinlichkeit, dass das Sample der Gesamtgruppe entspricht, bei einer Gesamtgruppe von 50 ein Sample von 44 erforderlich ist, bei einer Gesamtgruppe von 1000000 ein Sample von 384 (Bernard 1995: 79, Tabelle 4.3). Mathematisch Interessierte finden bei Bernard auch die Formel, mit der die Samplegröße berechnet werden kann (ibid., 78).

\subsection{Erhebungswege}

Wie werden die Fragen den Informanten bzw. dem ausgewählten Sample nun tatsächlich gestellt? Grundsätzlich können über die verwendeten Kommunikationswege drei Arten der Befragung unterschieden werden: a) mündliche Befragung durch den Interviewer; b) telefonische Befragung durch Interviewer; c) schriftliche Befragung, bei der die Informanten einen vorliegenden Fragebogen selbst ausfüllen. Für alle Erhebungsarten gilt, dass die Erhebung in einem möglichst kurzen Zeitraum durchgeführt werden sollte, um zu vermeiden, dass Ereignisse eintreten, die das Antwortverhalten der später Interviewten entscheidend beeinflussen.

\subsection{Direkte mündliche Befragung}

In vielen Fällen ergibt sich die Erhebungsweise schon aus der Art und dem Ort der Forschung.

In vielen Gebieten, in denen Ethnologen klassischerweise ihre Feldforschungen durchführen, sind andere Erhebungsweisen als die direkte mündliche Befragung durch einen Interviewer nicht möglich. Eine telefonische Befragung scheidet vielleicht aus, weil Telefonieren dort eine eher außergewöhnliche Kommunikationsform ist oder weil überhaupt nur wenige Menschen an ein Telefonnetz (soweit vorhanden) angeschlossen sind. Eine schriftliche Befragung ist vielleicht nicht möglich, weil viele der Informanten des Lesens und Schreibens nicht kundig sind.

Mündliche Interviews sind in der Regel zeitaufwändig. Das gilt nicht nur, wenn der Fragebogen sehr umfangreich ist, sondern auch weil man in der Regel nicht mit einem Fragebogen ,ins Haus fallen' und gleich wieder verschwinden kann, wenn alle Fragen abgehakt sind. Die mündliche Befragung ist in der Regel in einen ,Besuch ' eingebettet, und ein ,Besuch ' kann je nach kulturellem Kontext ein relativ lang andauerndes Ereignis sein. Bevor man zum Fragebogen kommt, muss man vielleicht verschiedene Begruißungsrituale absolvieren und ausführlich ,Smalltalk' führen - wobei small ein sehr irreführendes Adjektiv sein kann. Oft erfordert ein Besuch, dass der Besuchte, der sich dann als Gastgeber versteht, dem Besucher und Gast etwas zu Essen oder zu Trinken anbietet, was der Interviewer als Gast wiederum nicht ablehnen darf. Es kann also länger dauern, bis man überhaupt dazu kommt, den Fragebogen hervorzuholen. Und wenn er denn abgearbeitet ist, dann müssen vielleicht analog zur Ankunft diverse Abschiedsformalitäten eingehalten werden. Diese kommunikative Einbettung einer Befragung muss kein Nachteil sein, da man auch beim Gespräch vor und nach dem Interview zahlreiche Informationen erhalten kann. 
In einem solchen Kontext kann man also nicht davon ausgehen, dass ein Interview nur so viel Zeit erfordert, wie man etwa für das testweise Durchgehen der Fragen mit einem Probeinformanten benötig. Unter Umständen kann man sich glücklich schätzen, wenn man pro Tag auch nur zwei Interviews durchführen kann. Wenn man als Ethnologe für die Feldforschung ein Jahr Zeit hat, aber nicht das ganze Jahr mit standardisierten Interviews verbringen möchte, kann man sich ausrechnen, dass ein einzelner Forscher auf diese Art nicht sehr viele Personen befragen kann.

Hier ist der Einsatz von Forschungsassistenten sinnvoll. Wenn man einen Antrag auf Forschungsmittel stellt, sollte man gleich überlegen, welche Befragungen geplant sind, und ob es sinnvoll ist, Mittel für Assistenten zu beantragen. Wenn man Assistenten engagiert, muss man sie jedoch sehr gründlich auf ihre Aufgabe vorbereiten. Sie müssen beispielsweise die Fragen so gut verstehen, dass sie sie auf Nachfrage dem Interviewten erläutern können. Auch dürfen sie die Frage nicht auf eine suggestive Art und Weise stellen, die den Informanten schon eine Antwort in den Mund legt. In der Regel ist es sinnvoll, dass man nach einer theoretischen Anleitung mehrere Interviews gemeinsam mit den Assistenten durchführt, ihr Frageverhalten beobachtet und nach der Interviewsituation mit ihnen bespricht und korrigiert. Axinn et al. (1991) gehen ausführlicher auf das Training von Interviewern ein

\subsubsection{Telefonische Befragung}

Telefonische Befragungen assoziiert man eher mit Wahl- oder Marktforschung, aber sie kann auch in der Ethnologie sinnvoll sein. Das ist der Fall, wenn einerseits Telefone weit genug verbreitet sind, so dass man seine Informanten tatsächlich telefonisch erreichen kann, und wenn andererseits die Informanten so weit verstreut leben, dass man sie gar nicht alle zu einem Interview aufsuchen kann.

Telefonische Interviews lassen sich oft schneller erledigen als Interviews im direkten Gespräch, da man am Telefon häufig direkter ,zur Sache“ kommen kann und die sonst vielleicht obligatorische Tasse Kaffee beim Gespräch ausfällt. Gleichzeitig ist die Atmosphäre häufig unverbindlicher. Das kann eine gewisse Anonymität herstellen, in der sich manche Dinge leichter fragen und beantworten lassen als im persönlichen Gespräch. Genauso wie im persönlichen Gespräch sind auch beim Telefoninterview Nachfragen durch die Informanten möglich, Unklarheiten können also ausgeräumt werden. Eine Schwierigkeit bei Telefoninterviews besteht darin, überhaupt die Telefonnummern herauszufinden. Selbst in Deutschland, wo CD-ROMs oder Internetabfragen zur Verfügung stehen, ist das nicht immer leicht.

\subsubsection{Schriftliche Befragung}

Bei der schriftlichen Befragung wird dem Befragten der Fragebogen übergeben, den er dann selbständig, ohne Beteiligung eines Interviewers ausfüllt Das hat einige Nachteile. Nachfragen sind nicht möglich, der Interviewte interpretiert den Fragebogen auf seine Art, und diese Interpretation kann von dem, der die Fragebögen erstellt und verteilt hat, nicht nachvollzogen werden. Man weiß also nicht, ob die Fragen, richtig verstanden wurden. Der Forscher kann die Art des Verständnisses nicht aus Nebeninformationen, wie sie im Gespräch selbstverständlich sind, schließen. Diese Schwierigkeit erfordert besondere Sorgfalt bei der Erstellung von Fragebögen. Fragen müssen besonders einfach und eindeutig formuliert werden. Ein schriftlicher Fragebogen muss außerdem von einem Anschreiben begleitet werden, das Sinn und Zweck der Befragung erläutert, Datenschutz und Anonymität zusichert und auch eine Kontaktadresse für die Informanten nennt, falls sie doch weitergehende Fragen haben.

Schriftliche Befragungen sind in der Marktforschung sehr üblich. In der Ethnologie können sie dann sinnvoll sein, wenn man ein großes Sample befragen möchte und dazu die Möglichkeiten direkter oder telefonischer Befragung nicht ausreichen. Häufig wird eine schriftliche Befragung per Post durchgeführt. Dies ist sinnvoll, da man dann kontrollieren kann, wer einen Fragebogen erhält und wer nicht. Natürlich kann man Fragebögen auch selbst verteilen oder in einem Schneeballsystem verteilen lassen. Dann fehlt aber die Kontrolle über ihre Verteilung und es ist entsprechend schwieriger, die Aussagekraft der Ergebnisse einzuschätzen.

Der postalische Versand von Fragebögen setzt zwei wichtige Ressourcen voraus. Dies sind einerseits die Adressen der Informanten und andererseits ausreichende Mittel für Porto, Rückporto und eventuell notwendige Erinnerungsschreiben. Aufgrund des gestiegenen Bewusstseins für Datenschutz ist es allgemein schwieriger, Adressen zu bekommen. Für allgemeine, repräsentative Surveys kann man sich mit dem Telefonbuch behelfen und nach einer $\mathrm{Zu}-$ fallsmethode Adressen heraussuchen. Dies hilft aber nicht weiter, wenn man ein bestimmtes Segment einer Bevölkerung befragen will. Hier muss man versuchen, über Einrichtungen oder Vereine, mit denen diese Gruppe zu tun hat, weiter zu kommen. Während ich diesen Beitrag schreibe, bereite ich eine postalische Erhebung unter den Mitgliedern alevitischer Vereine in Hamburg vor. Um die Adressen zu bekommen, bin ich auf die Kooperation dieser Vereine angewiesen. Diese Kooperation ist nur möglich, wo ein größeres Vertrauen aufgrund guter Kontakte besteht. Die Nutzung deartiger Mittlerinstitutionen schränkt natürlich die Gültigkeit der Ergebnisse ein. So bekom- 
me ich mit meiner Erhebung keine Informationen über Aleviten in Hamburg, sondern lediglich über die Mitglieder alevitischer Vereine in Hamburg. Dies ist ein wichtiger Unterschied, da vermutlich nur solche Aleviten Mitglieder eines Vereins werden, für die es eine relativ große Bedeutung hat, Alevit zu sein. Es ist anzunehmen, dass sie viele Fragen anders beantworten als solche Aleviten, denen das Alevitentum nicht viel bedeutet.

Eine Schwierigkeit bei postalischen Befragungen besteht darin, dass die Antwortquote in der Regel geringer ist als in der persönlichen oder telefonischen Interviewsituation. Man wirft eben leichter einen Fragebogen ins Altpapier, als dass man ein Interview ablehnt, wenn man direkt angesprochen wird. Tipps, wie sich die Antwortquote bei postalischen Befragungen erhöhen lässt, finden sich bei Dillman (1978, 1983).

\subsection{Auswertung}

Wir haben nun also einen Fragebogen erstellt, ein Sample ausgewählt und die Befragung durchgeführt. Die ausgefüllten Fragebögen stapeln sich im Zelt neben dem Schlafsack des Forschers oder auf dem heimischen Schreibtisch. Was fängt man damit nun an?

Das Thema dieses Bandes ist die Datenerhebung. Verfahren zur Datenauswertung würden ein eigenes Buch füllen. Da es aber unbefriedigend wäre, auf die Auswertung der erhobene Daten überhaupt nicht einzugehen, sollen hier wenigstens einige einführende Informationen gegeben werden. Zum weitergehenden Studium verweise ich auf den Beitrag von Handwerker und Borgatti (2000), der auch zahlreiche weiterführende Literaturangaben enthält.

Die Auswertung der erhobenen Daten geschieht heute in der Regel mit entsprechenden Computerprogrammen, wie z.B. dem Statistical Package for the Social Sciences (SPSS). Das befreit den Forscher davon, selbst komplizierte Rechnungen durchzuführen. Die sinnvolle Benutzung derartiger Programme setzt aber Statistikkenntnisse voraus, damit man die Operationen des Programms nachvollziehen kann und weiß, welche Operationen mit welchen Daten sinnvoll durchgeführt werden können. An dieser Stelle kann ich keinen Crashkurs in Statistik geben, nur einige Grundbegriffe sollen vermittelt werden. Eine gute Einführung in die Statistik, die gleichzeitig die Arbeit mit SPSS vorstellt, bieten Diehl und Staufenbiel (2001).

\subsubsection{Typen von Variablen}

Mit den Fragen eines Fragebogens werden verschiedene Variablen erhoben. Diese Variablen können sehr unterschiedlichen Charakter haben. Dichotome Variablen können lediglich zwei Zustände annehmen. So wird in zahlreichen Gesellschaften die Variable ,Geschlecht" entweder die Ausprägung ,weiblich ' oder , männlich' haben. Dichotom sind auch alle Variablen, die mit einer $\mathrm{Ja} / \mathrm{Nein}-F r a g e$ erhoben werden. Dichotome Variablen sind gleichzeitig nominale Variablen. Sie erfassen Eigenschaften, die an sich keine Quantitäten wieder geben. Männlich ist nicht mehr als weiblich. Nominale Variablen sind nicht immer dichotom, die Variablen können auch mehr Zustände annehmen können. Betrachten wir die Variable ,Familienstand' mit vier Ausprägungen: ledig, verheiratet, geschieden, verwitwet. Diese Variable ist polytom. Nominale Variablen geben Qualitäten wieder und sind dadurch charakterisiert, dass man lediglich die Häufigkeit ihrer Zustände feststellen, aber keine weitergehenden Rechenoperationen mit ihnen durchführen kann. So kann man auszählen, wieviele Informanten ledig oder verwitwet sind, es ist aber unmöglich, etwa einen Mittelwert der Ausprägungen angeben. Das Gleiche gilt für ordinale Variablen, die Rangfolgen erfassen, wie etwa der Schulabschluss oder der soziale Status. Hier ist zwar ein ,mehr' oder, weniger impliziert, das Abitur gilt eben , mehr' als ein Hauptschulabschluss. Aber auch hier können weder Mittelwerte gebildet noch der Abstand zwischen zwei Merkmalsausprägungen quantifiziert werden.

Dies ist bei numerischen Variablen anders, die tatsächlich Quantitäten angeben. Das Alter oder die Haushaltsgröße sind numerische Variablen, die keine Eigenschaften erfassen, sondern Mengen und daher Rechenoperationen erlauben. Numerische Variablen können eine diskrete Ausprägung haben und nur ganzzahlige Ergebnisse liefern, wie etwa die Zahl der Kinder in einem Haushalt. Oder sie sind kontinuierlich und können im Prinzip jede Zahl annehmen, was beispielsweise für die Größe von Landbesitz oder das Gewicht der Ernte zutrifft.

\subsubsection{Die Codierung der Daten}

Bevor quantitativ erhobene Daten ausgewertet werden können, muss man sie codieren. Dabei wird jeder möglichen Merkmalsausprägung einer Variablen ein Code zugeordnet, in der Regel eine Zahl. Die Codierung wird in einem Codebuch festgehalten, damit auch im Nachhinein die Bedeutung der Codes wieder expliziert werden kann. Für die Variable ,Familienstand ' kann etwa festgelegt werden: ledig $=1$; verheiratet $=2$; geschieden $=3$; verwitwet $=4$. 
In der Datentabelle werden dann nur noch die entsprechenden Codes notiert. Man darf sich dabei nicht durch die Tatsache verwirren lassen, dass auch Nominalvariablen mit Ziffern ausgedrückt werden. Mit diesen Ziffern kann nicht gerechnet werden! Um das zu verhindern, muss im Codebuch unbedingt festgehalten werden, welchem Typ die jeweilige Variable enstpricht, d. h. ob es sich um eine nominale, ordinale oder numerische Variable handelt. Neben der exakten Beschreibung einer Variablen mit den möglichen Merkmalsausprägungen und dem Typus enthält ein Codebuch normalerweise auch die laufende Nummer der Variablen (die meistens der Nummer der Frage im Fragebogen entspricht) sowie eine Abkürzung als Namen der Variablen.

Im Abschnitt über die Konstruktion von Fragebögen wurde zwischen offenen und geschlossenen Fragen unterschieden. Geschlossene Fragen, bei denen die Antwortkategorien (die vollständig und disjunkt sein müssen) schon vorgegeben sind, erleichtern die Codierung. Man kann gleich auf dem Fragebogen hinter jedem Kästchen zum Ankreuzen den zugeordneten Code angeben. Die Codierung offener Fragen ist schwieriger. Sie kann erst nach der Befragung vorgenommen werden, da vorher gar nicht bekannt ist, welche Antworten vorkommen werden. In diesem Fall müssen aus den Antworten sinnvolle Kategorien gebildet werden, denen man dann die Codes zuordnet.

Numerische Daten müssen nicht notwendigerweise codiert werden. Bei der Variable ,Alter" kann einfach das Alter als Anzahl der Lebensjahre in die Datentabelle eingetragen werden. Es ist aber unbedingt erforderlich, dass die Einheit im Codebuch festgehalten wird, also etwa, dass das Alter in Jahren angegeben wird. Es kann auch sein, dass man numerische Daten nicht als solche weiterverarbeiten will, sondern dass sie in Gruppen zusammen gefasst werden. So ist es vielleicht sinnvoll, die Variable ,Einkommen " nicht in absoluten Zahlen festzuhalten, sondern in Tausendergruppen, etwa , bis $1000 €$ ', , 1001 bis $2000 €$ ', ,2001 bis $3000 €^{\prime}$ und so weiter. Dann wird jeder Gruppe ein Code zugeordnet. In solchen Fällen muss man sich überlegen, ob gleich bei der Datenerhebung nur danach gefragt werden soll, in welche Gruppe das Einkommen der Informanten fällt, oder ob das exakte Einkommen erhoben wird und man erst im Nachhinein Gruppen bildet. Dabei ist es wichtig zu beachten, dass Daten, die nur in Gruppen erhoben werden, nicht wieder in exakte Angaben zurückverwandelt werden können, auch wenn das zu einem späteren Zeitpunkt der Analyse sinnvoll wird. Man hält sich also mehr Möglichkeiten der Analyse offen, wenn Daten erst nachträglich in Gruppen zusammengefasst und codiert werden.
Häufig liegen nicht von allen Informanten Daten zu allen Variablen vor, etwa weil ein Informant eine Frage nicht beantworten kann oder will. Derartige fehlenden Daten werden gesondert codiert, damit sie bei der Analyse als solche erkannt und behandelt werden können.

\subsubsection{Die Datentabelle}

Die codierten Daten können nun in eine Datentabelle oder Matrix eingetragen werden. Die Datentabelle ist so organisiert, dass alle Angaben zu einem Informanten sich in einer Zeile befinden. Diese Daten bilden einen Datensatz. Wenn man hundert Interviews geführt hat, liegen also hundert Datensätze vor. Die Variablen sind in Spalten angeordnet. Auch Statistikprogramme wie SPSS arbeiten mit solchen Datentabellen. Dabei müssen die Spalten jeweils für die entsprechende Variable eingerichtet werden. Der Spalte wird der Name der Variable zugeordnet, die Art der Variablen und ihre Codierung werden angeben. Die erste Spalte der Datenmatrix enthält normalerweise die laufende Nummer des Datensatzes (d. h. die Nummer des Informanten). Daran schließen sich Spalten zu allen Variablen an.

Mit Statistikprogrammen ist es kein Problem, auch große Datentabellen zu verwalten - und Datentabellen werden sehr schnell sehr groß. Wenn man hundert Informanten befragt hat und von jedem Informanten sechzig Variablen erhoben hat, dann umfasst die Matrix schon 6000 Felder.

Die Eingabe der Daten ist eine ermüdende Tätigkeit, besonders bei zahlreichen und großen Datensätzen. Umso wichtiger ist es, dass die Dateneingabe mit großer Sorgfalt geschieht, denn Fehler bei der Dateneingabe gehören zu den häufigsten Fehlerquellen bei der Auswertung. Es ist daher wichtig, die Daten nach der Eingabe noch einmal zu überprüfen.

\subsubsection{Univariate Statistik}

Die quantitative Auswertung von Daten lässt sich in zwei große Bereiche unterteilen. Der erste betrifft die jeweils einzelnen Variablen und beschreibt die für sie erhobenen Daten. Da jede Variable für sich betrachtet wird, spricht man von univariater oder auch von deskriptiver Statistik. Der zweite Bereich betrifft die Analyse von Relationen zwischen verschiedenen Variablen, mit denen spezifische Hypothesen getestet werden. Ich gehe in diesem Beitrag lediglich auf die univariate Statistik ein. 
Drei Bereiche univariater Statistik können unterschieden werden: Häufigkeitsverteilung, zentrale Tendenz und Streuung. Zur Feststellung der Häufigkeitsverteilung wird errechnet, wie oft jede Merkmalsausprägung einer Variablen im Sample vorkommt. Dazu legt man eine Häufigkeitstabelle an, in der die Häufigkeit jedes Zustandes normalerweise sowohl in absoluten Zahlen als auch in Prozenten angegeben wird. Oft werden zusätzlich zu den Angaben zu den einzelnen Merkmalsausprägungen auch jeweils die kumulierten Angaben (meist in Prozenten) errechnet, damit man auf einen Blick die gemeinsame Häufigkeit mehrerer Merkmalsausprägungen sehen kann. So kann man in der folgenden Tabelle der Anzahl von Kindern in einem Haushait auf einen Blick erkennen, dass in $65,7 \%$ der Haushalte höchstens zwei Kinder leben. Konventionell wird zu der Tabelle die Größe des Samples (n) angegeben.

Häufigkeitstabelle: Zahl der Kinder im Haushalt

$\begin{array}{cccc}\text { Zahl der Kinder } & \text { Häufigkeit } & \text { Prozent } & \text { kumulierte Prozente } \\ 0 & 5 & 14,3 & 14,3 \\ 1 & 7 & 20,0 & 34,3 \\ 2 & 11 & 31,4 & 65,7 \\ 3 & 8 & 22,9 & 88,6 \\ 4 & 3 & 8,6 & 97,1 \\ 5 & 1 & 2,9 & 100,0\end{array}$

$\mathrm{n}=35$

Maße der zentralen Tendenz geben die Mittelwerte an. Das arithmetische Mittel ist sicherlich das bekannteste $\mathrm{Ma}$ der zentralen Tendenz, es errechnet sich aus der Summe der erhobenen Werte geteilt durch die Anzahl der Fälle. Im Fall der Anzahl der Kinder im Haushalt ergibt sich so ein arithmetisches Mittel von zwei Kindern. Das arithmetische Mittel kann nur für numerische Variablen errechnet werden. Ein weiteres Maß ist der Median. Der Median unterteilt die erhobenen Werte so, dass er genau in der Mitte liegt, d.h. die eine Hälfte der aufgetretenen Werte liegt , vor' dem Median, die andere Hälfte folgt ,nach“ dem Median. Ein weiteres Maß der zentralen Tendenz ist der Modus oder Modalwert. Er ist der im Sample am häufigsten vorkommende Wert. Es können bei einer Messung auch mehrere Modi vorkommen, wenn mehrere Werte gleich häufig gemessen werden.

Mit der Angabe von Mittelwerten allein ist ein Sample unvollständig beschrieben. Darauf weist ein alter Statistikerwitz hin: Ein Statistiker hat die rechte Hand im Eisfach und die linke auf einer heißen Herdplatte. Er kommentiert: ,Im Mittelwert eine angenehme Temperatur!' Maße der Streuung liefern Informationen über die Verteilung der Werte in einem Sample. Hier ist zunächst die Spannweite zu nennen, die sich aus der Differenz zwischen dem größten und dem kleinsten gemessenen Wert ergibt. In die Spannweite gehen nur zwei der gemessenen Werte ein, die Standardabweichung berücksichtigt dagegen alle Werte. Sie errechnet sich, indem man die Wurzel aus der durchschnittlichen quadrierten Abweichung vom Mittelwert bildet. Die Standardabweichung ist ein $\mathrm{Maß}$ für die Homogenität oder Heterogenität der Meßwerte: Werden nur identische Werte gemessen, ist die Standardabweichung null.

Mit Häufigkeiten, Maßen der zentralen Tendenz und Maßen der Streuung lassen sich die Erhebungsergebnisse zu den einzelnen Variablen beschreiben. Wichtig ist, dass es hier immer nur um einzelne Variablen geht, nicht um Beziehungen und Zusammenhängen zwischen mehreren Variablen.

\subsection{Schluss}

Quantitative Methoden werden mehr mit Soziologie assoziiert als mit Ethnologie. Dass auch die Ethnologie ohne Quantifizierung nicht auskommt, hat dieser Beitrag gezeigt. Die Verfahren, die Soziologen und Ethnologen bei quantitativen Erhebungen anwenden, sind identisch. Aber es gibt trotzdem Unterschiede: Da Ethnologen häufiger in anderskulturellen Kontexten arbeiten, müssen sie bei der Erstellung von Fragebögen, bei der Auswahl von Kategorien und der Formulierung der Fragen noch größere Sorgfalt walten lassen als die Kollegen aus der Soziologie. Und schließlich kommt in der Ethnologie etwas kaum vor, was in der Soziologie recht häufig ist: dass eine Forschung allein mit quantitativen Daten operiert. Man mag das - etwa aus der Perspektive der empirischen Sozialforschung - für einen Mangel an Exaktheit halten, tatsächlich drückt sich darin aber die Erkenntnis aus, dass sich nicht alle Aspekte menschlicher Lebenswelten sinnvoll in Zahlen erfassen lassen. Ethnologische Forschung wird daher nie ohne eine Kombination verschiedener Erhebungsmethoden auskommen.

\subsection{Literatur}

Axinn, William G.; Thomas E. Fricke; Arland Thornton

1991 The Microdemographic Community-Study Approach. In: Sociological Methods \& Research 20: 187-217.

Bernard, H. Russel

1995 Research Methods in Anthropology. Walnut Creek. 
Brislin, R. W.

1986 The Wording and Translation of Research Instruments. In: Lonner, W. J.; J. W. Berry (Hg.): Field Methods in Cross-cultural Research: 137-164. Thousand Oaks.

Diehl, Joerg M.; Thomas Staufenbiel

2001 Statistik mit SPSS. Eschborn.

Dillman, D. A.

1978 Mail and Telephone Surveys: The Total Design Method. New York.

1983 Mail and Other Self-administered Questionnaires. In: Rossi, P. H.; J. D. Wright; A. B. Anderson (Hg.): Handbook of Survey Research. New York, Academic Press: $359-378$.

Evans-Pritchard, Edgar Evan

[1940] 1969 The Nuer. Oxford.

Handwerker, W. Penn; Stephen P. Borgatti

2000 Reasoning with Numbers. In: Bernard, H. Russel (Hg.): Handbook of Methods in Cultural Anthropology: 549-594. Walnut Creek.

Headland, Thomas N.; Kenneth L. Pike; Marvin Harris (Hg.)

1990 Emics and Etics. The Insider/Outsider Debate. Newbury Park.

Schweizer, Thomas

2000 Epistemology. The Nature and Validation of Anthropological Knowledge. In: Bernard, H. Russel (Hg.): Handbook of Methods in Cultural Anthropology: 39-87. Walnut Creek.

Sobo, Elisa J.; Victor C. De Munck

1998 The Forest of Methods. In: De Munck, Victor C.; Elisa J. Sobo (Hg.): Using Methods in the Field. A Practical Introduction and Casebook: 13-37. Walnut Creek.

Weller, Susan C.

1998 Structured Interviewing and Questionnaire Construction. In: Bernard, H. Russel (Hg.): Handbook of Methods in Cultural Anthropology: 365-409. Walnut Creek. 